\title{
The CMS inner tracker - transition from LHC Run I to Run II and first experience of Run II
}

\author{
Benedikt Vormwald* $\dagger$ \\ University of Hamburg, Germany \\ E-mail: benedikt.vormwaldecern.ch
}

\begin{abstract}
The CMS silicon pixel and strip trackers provide high efficiency charged particle reconstruction and superb momentum resolution over three decades in energy, and thus play a key role in the CMS physics program. The readiness of the silicon tracking detectors for LHC Run II data taking is presented in this paper. In light of improvements to the tracker operating environment and repairs of defective pixel channels during the first LHC long shutdown, the Run II tracker is expected to have a larger yield of active channels than during Run I and to continue to perform well at the foreseen luminosities.
\end{abstract}

The European Physical Society Conference on High Energy Physics

22-29 July 2015

Vienna, Austria

\footnotetext{
${ }^{*}$ Speaker.

${ }^{\dagger}$ for the CMS collaboration
} 


\section{Introduction}

The tracker system is the centerpiece of the Compact Muon Solenoid (CMS experiment) [1], which is one of the two general-purpose detectors at the Large Hadron Collider (LHC) at CERN. It consists of a highly granular silicon pixel detector in the center and a silicon strip detector in the outer region. The pixel detector features three barrel layers (BPIX) placed at a distance of $r=4.4-7.3 \mathrm{~cm}$ from the interaction point as well as two forward disks (FPIX) on each side ${ }^{1}$. In total, the pixel detector has 66 million pixels of a size of $100 \times 150 \mu \mathrm{m}^{2}$. The strip detector has 10 barrel layers and $9+3$ forward disks on each side with in total 9.3 million strips featuring a strip pitch of $83-205 \mu \mathrm{m}$. Figure 1 shows a longitudinal cross section of the upper half of the tracker.

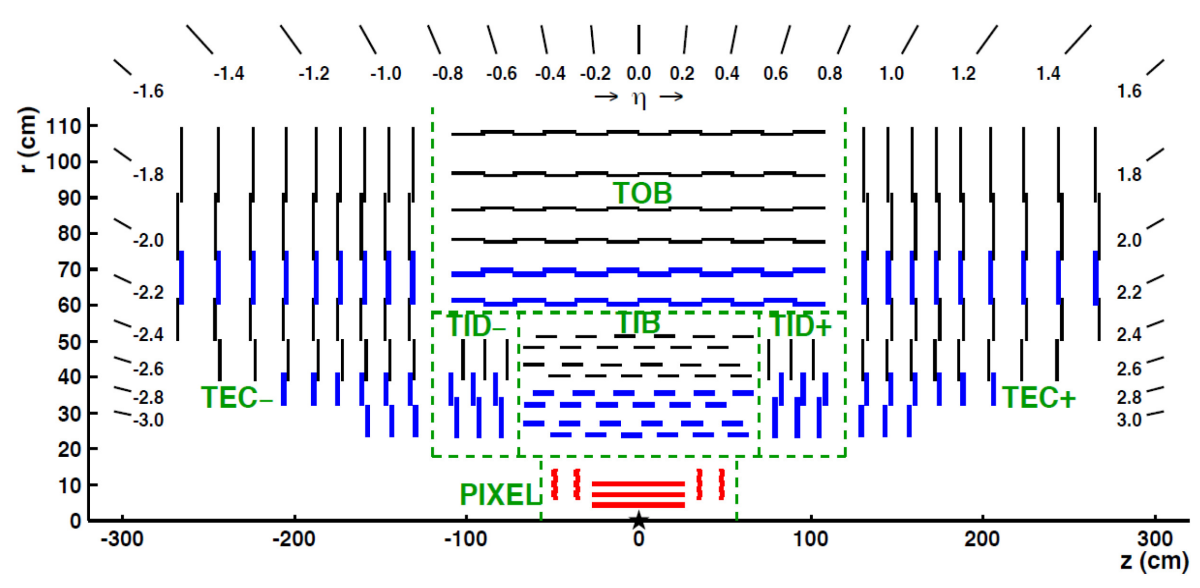

Figure 1: Schematic view of the upper half of the CMS inner tracker.

The CMS tracker is the largest all-silicon central tracker ever built, with a total sensitive area of about $200 \mathrm{~m}^{2}$. Both the pixel and the strip detectors are designed for a hit coverage in pseudorapidity of up to $|\eta|<2.5$.

\section{The CMS Tracker during LHC Run I}

\subsection{Tracker Performance}

The CMS tracker has shown an excellent performance during the LHC Run I (2010-2012). The average hit finding efficiency in the pixel detector has been above 99\% in all the layers and disks (Figure 2 (left)), with layer one showing the largest inefficiency due to the proximity to the interaction point. For very high instantaneous luminosities of $7000 \mu \mathrm{b}^{-1} \mathrm{~s}^{-1}$ an efficiency drop larger than $2 \%$ has been measured in the first pixel layer as also predicted from simulations of the read-out chip. This is the main motivation for the CMS pixel detector Phase I upgrade program [2].

Figure 2 (middle) depicts the effects of radiation damage on the measured $r-\phi$ hit resolution in the second pixel layer. A slight degradation from $8.2 \mu \mathrm{m}$ to $9.6 \mu \mathrm{m}$ has been observed.

\footnotetext{
${ }^{1}$ Coordinates within CMS are described in a right-handed coordinate system $(x, y, z)$ with the interaction point at the origin of the coordinate system. $x$ points into the center of LHC and $y$ upwards. $r=\sqrt{x^{2}+y^{2}}$ describes the distance to the origin in the transversal detector plane. $\eta$ is the pseudo-rapidity and can be related to polar angle with respect to the beam axis.
} 

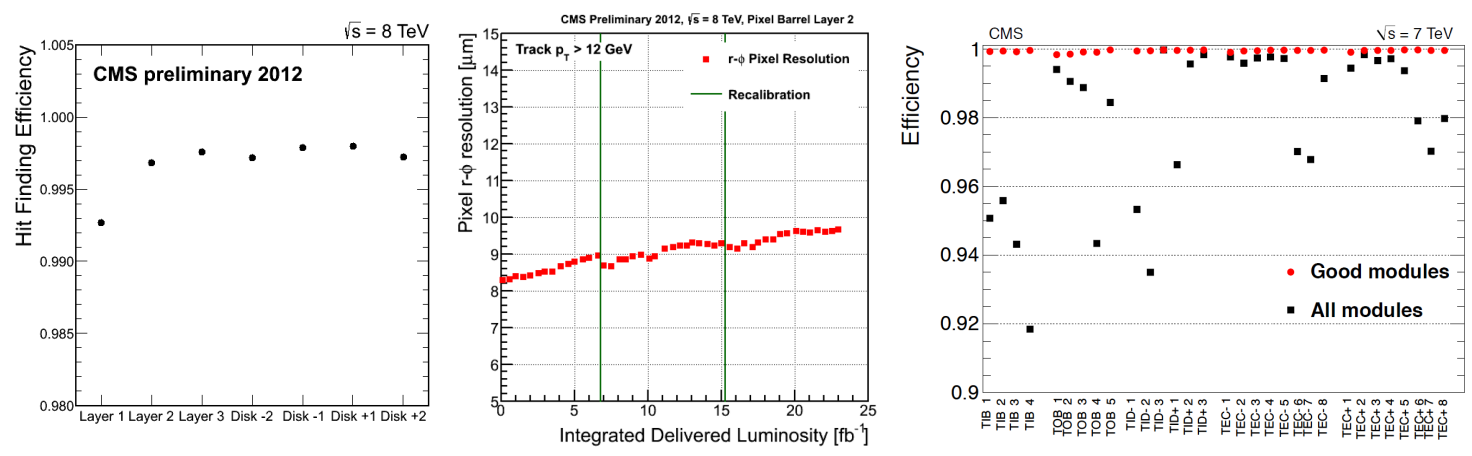

Figure 2: Performance of the CMS pixel and strip detector during LHC Run I. Left: Hit finding efficiency of the pixel detector. Middle: Pixel hit resolution. Right: Hit finding efficiency of the strip detector.

Figure 2 (right) shows the hit finding efficiency for the strip detector in the different parts of the detector. When considering only good modules (red data points), which did not suffer from a general malfunction (see also Section 2.2), the module efficiency is better than $99.5 \%$.

\subsection{Detector Status at the End of LHC Run I}

At the end of LHC Run I, the number of operational detector channels in the FPIX detector was 92.2\% as one can deduce from Figure 3 (upper row). The main reason for this rather large inactive detector fraction was a broken optical read-out connection and digitization problems with distorted analog signal output. In the BPIX detector, $97.7 \%$ of the channels were functional as illustrated in Figure 3 (lower row). Most of the faulty channels showed broken wire-bond connections. The repair of the pixel detector was one important activity during the first LHC long shutdown.
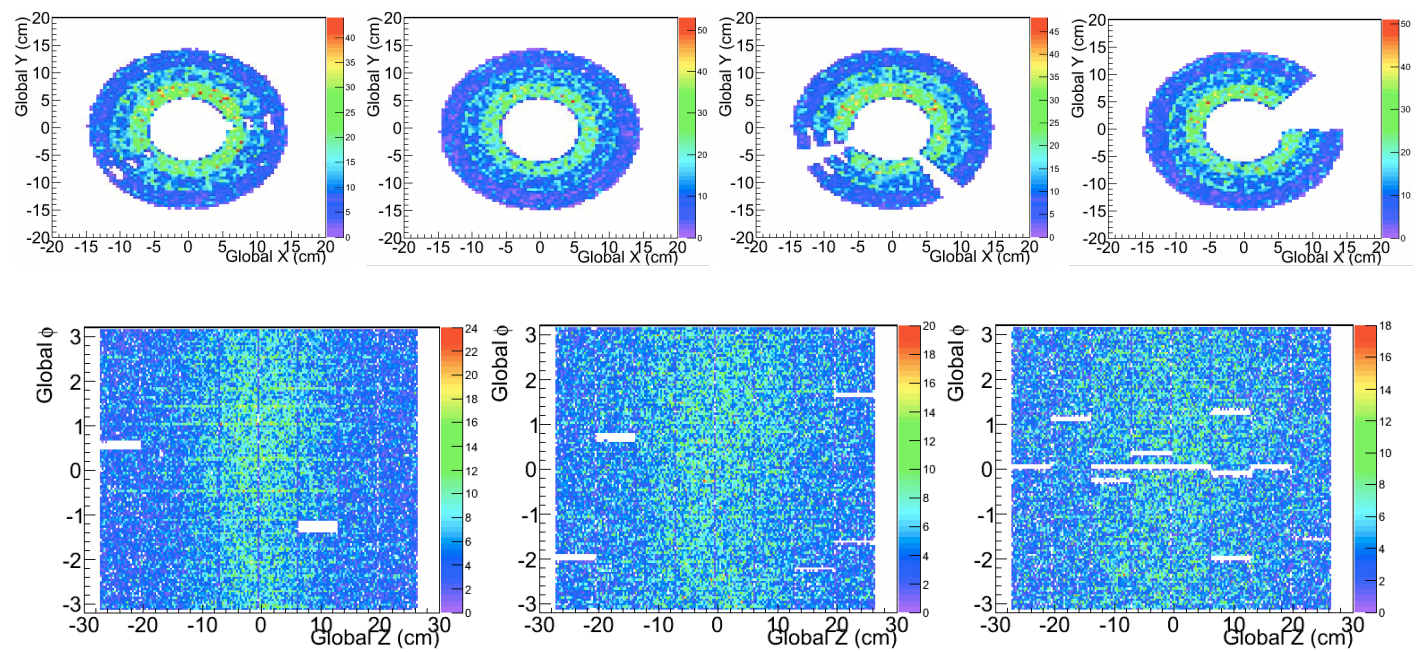

Figure 3: Status of the CMS pixel detector at the end of LHC Run I. Upper row: four forward pixel disks. Lower row: three barrel pixel layers.

In the silicon strip detector, $97.5 \%$ of the detector channels were still active at the end of LHC Run I, as shown in Figure 4. Most of the problems affect groups of modules (e.g. problems in 
control token ring networks or low/high voltage distribution). Due to the inaccessibility of the outer tracker, no major repairs could be pursued during LHC long shutdown 1.

$+\mathbf{z}$

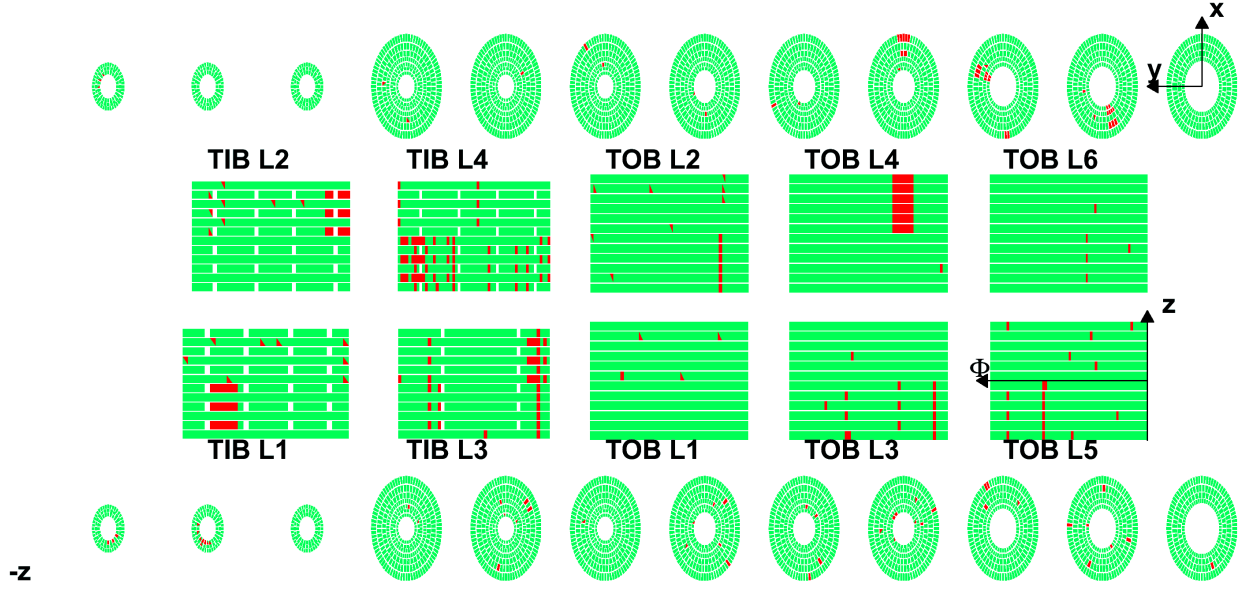

Figure 4: Status of the CMS strip detector at the end of LHC Run I. Red areas mark non-functioning modules.

\section{Maintenance during LHC Long Shutdown I}

\subsection{Repair of the Pixel Detector}

One of the design principles of the CMS pixel detector has been the possibility of fast access to the detector. This allowed to extract the pixel detector from CMS in summer of 2013 for repair. The detector was stored in temperature and humidity controlled boxes in a clean room at the surface. During the time at the surface, almost all faulty FPIX channels and half of the faulty BPIX channels could be repaired.

From December 2014 until January 2015, the repaired detector was re-installed and re-commissioned. Due to the successful repair campaign only $0.04 \%$ of the FPIX channels and $<1 \%$ of the BPIX channels were inactive at the beginning of Run II (2015-2018), which is a significantly improved situation compared to Run I.

First alignment studies (see also Section 4) show that especially the forward pixel detector in negative $z$-direction is shifted in the order of a few millimeters in $z$ with respect to the alignment during LHC Run I, as shown in Figure 5. The detector geometry has been updated accordingly.

\subsection{Improvements of Tracker Operation Conditions}

During LHC Run I, the CMS tracker was operated at a temperature of $+4{ }^{\circ} \mathrm{C}$. A safe operation at lower temperatures was not possible during Run I because of too high dew-points in the tracker bulkhead region and in the service channels shown in Figure 6 (left). A lower temperature, however, is desirable, because the leakage currents in silicon drop significantly ( $50 \%$ for each $\Delta T=-7 \mathrm{~K}$ ). In connection with radiation damage, the high temperature would bring the tracker power supplies to their limits in the future. For this reason, a large effort was made to improve the humidity situation 


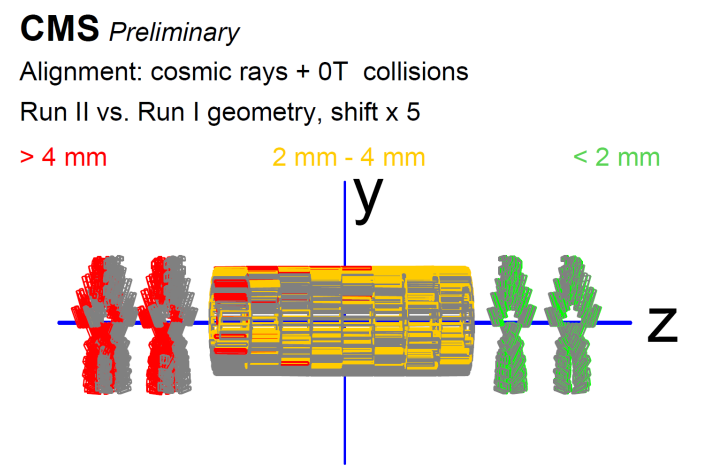

Figure 5: Pixel detector geometry after re-installation (colored) in comparison to the geometry during LHC Run I (grey). The color scheme indicates the deviation of the position between the two setups.

in the tracker volume: On the one hand, a new membrane-based dry-gas plant outputting ten times the amount of dry-gas of the old system has been installed. On the other hand, continuous sealing from the bulkhead to the CMS solenoid has been achieved as visible for instance on the photo in Figure 6 (right), which shows the front view onto the bulkhead in the opened CMS detector.
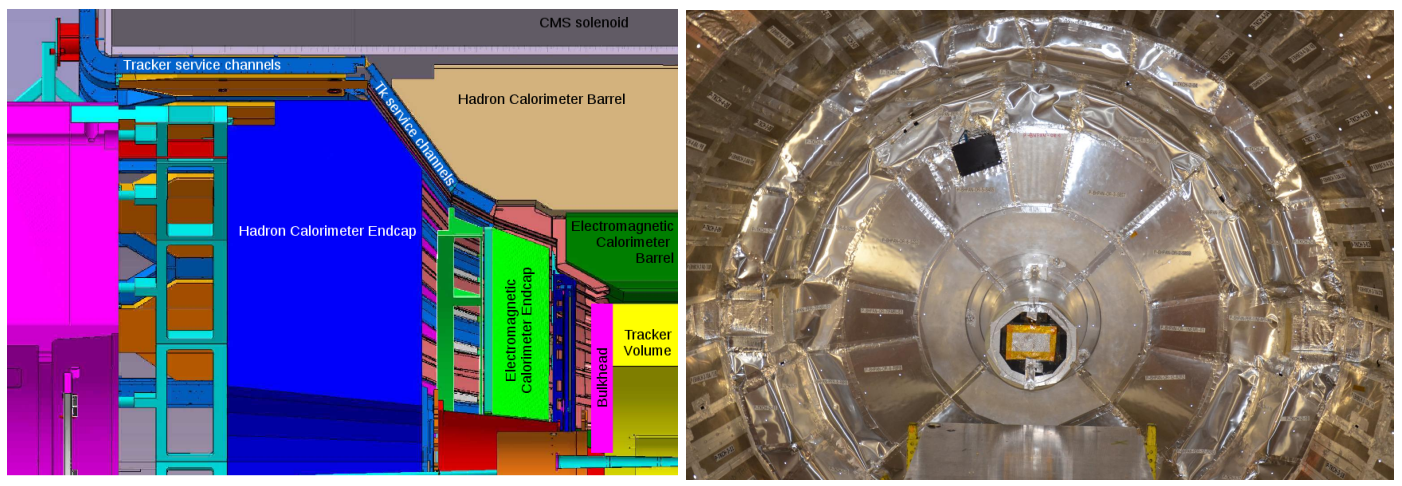

Figure 6: Left: Schematic drawing of the different detector parts and service channels in the endcap region of CMS. Too large dew-points in the tracker service channels and the bulkhead region prevented the tracker to be operated at the foreseen design temperature during LHC Run I. Right: Photo of the improved sealing of the CMS tracker volume.

For better monitoring of the tracker operation conditions, additional sensors have been installed: Fiber Optic Sensors (FOS) are installed for in-situ temperature and humidity monitoring [4]. Those sensors are radiation hard and will survive the foreseen lifetime of the tracker $\left(500 \mathrm{fb}^{-1}\right)$. Additionally, there is an independent sniffer system with a total of 26 lines analyzing gas from inside and outside the tracker volume in the CMS service cavern. As a third system, Arduino based in-situ temperature and humidity sensors are in place, which will not withstand the harsh radiation conditions during LHC operation, but gave valuable input for sensor cross-checks during commissioning of the new systems. In a cold run test, the design temperature of the CMS strip detector of $-20^{\circ} \mathrm{C}$ could be reached with dew-points below $-30^{\circ} \mathrm{C}$ throughout the entire tracker volume including the bulkhead region and service channels.

For LHC Run II, the operating temperature of the pixel detector has been set to $-10^{\circ} \mathrm{C}$. The strip detector is operated at a temperature of $-15^{\circ} \mathrm{C}$ instead of the design temperature of $-20^{\circ} \mathrm{C}$. 
This decision is based on a study of the increase of the final depletion voltage at the end of the LHC Phase-1 for an increased temperature during Run II with respect to running all the time at the original design temperature. Figure 7 shows the change of the depletion voltage at the end of the lifetime of the strip detector comparing the two temperature scenarios. The impact has been found to be small (about $5-10 \mathrm{~V}$ compared to a nominal depletion voltage of $300-400 \mathrm{~V}$ at $500 \mathrm{fb}^{-1}$ ) and, thus, the technically less demanding temperature of $-15^{\circ} \mathrm{C}$ has been chosen.

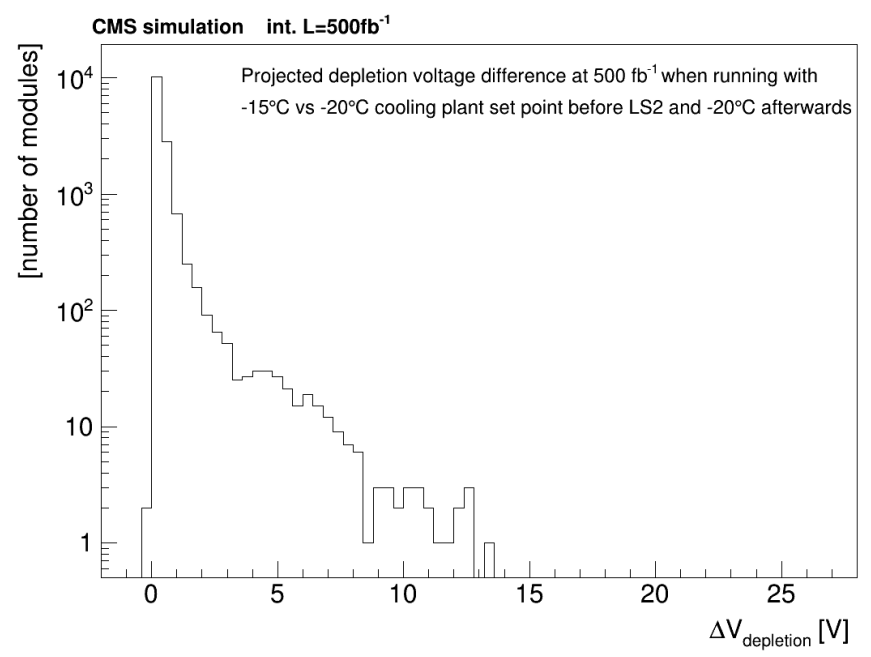

Figure 7: Difference of depletion voltage at the end of the lifetime of the strip detector for two different temperature scenarios. The found differences are negligible compared to the absolute depletion voltage of $300-400 \mathrm{~V}$ at $500 \mathrm{fb}^{-1}$.

\section{CMS Tracker Alignment}

In dedicated cosmic runs and from first collision data, new tracker alignment constants have been determined and cross-checked at the start of LHC Run II. One method to validate the detector geometry is the "Cosmic Track Splitting Validation". In this method, a cosmic track gets split into two tracks at the point of closest approach to the detector origin. The two half-tracks are refitted with the alignment constants under consideration.

Systematic deviations between the resulting track parameters of the two half-tracks would indicate biases and misalignments. Figure 8 shows as two examples the difference between the fitted transverse momentum $p_{T}$ with magnetic field switched on (left) and the fitted longitudinal impact parameter $d_{z}$ without magnetic field (right). No misalignment and bias can be observed. The comparison with Monte-Carlo simulations (red line) demonstrates that the tracker has almost reached its design resolution. The Run I geometry indicated by the blue data points is clearly not valid anymore for Run II data. Further details on the offline tracker performance are presented in [5].

\section{CMS Pixel Pilot System}

During the first LHC long shutdown, eight modules of the pixel Phase I upgrade program 

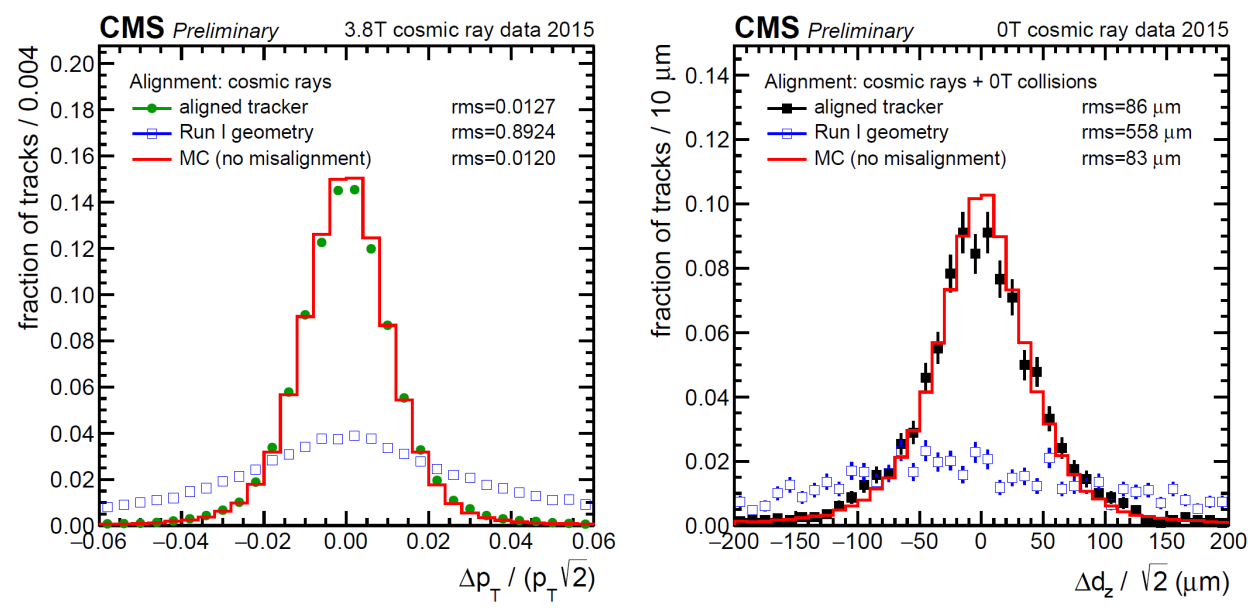

Figure 8: Validation of new tracker geometry with cosmic data with magnetic field switched on (left) and off (right).

have been installed on a third disk of the forward pixel detector. In the pixel Phase I upgrade at the end of 2016, the entire pixel detector will be replaced by a new detector. This is necessary in order to address the expected performance degradation of the current pixel detector in the harsher environment of LHC Run III (2021-2023). The status and recent developments of the Phase I pixel detector upgrade are presented in [3]. The new modules feature an improved digital token bit manager (TBM), which controls the read-out of a full module, as well as 16 improved digital read-out chips (ROCs) per module. The pilot system is fully embedded in the global CMS DAQ environment and, thus, allows to develop and exercise the full readout chain for the pixel Phase I upgrade. It further offers the unique opportunity to study already now the ROC and TBM performance under real LHC conditions. The pixel Phase I detector will be installed during the year-end technical stop 2016/2017 and the pilot system will ensure a smooth detector commissioning.

\section{Conclusions}

The CMS tracker has been successfully re-commissioned after many maintenance actions that took place during the first LHC long shutdown. For the pixel detector many broken channels could be repaired and the overall tracker operation conditions are now improved. First alignment and performance studies show that the re-commissioned detector is well under control such that it is very well suited for the LHC Run II.

\section{References}

[1] S. Chatrchyan et al. [CMS Collaboration], The CMS experiment at the CERN LHC, JINST 3, S08004 (2008).

[2] CMS Collaboration, CMS Technical Design Report for the Pixel Detector Upgrade, CERN-LHCC-2012-016 (2012).

[3] B. Vormwald, CMS Tracker Upgrades: R\&D Plans, Present Status and Perspectives, EPS-HEP 2015 proceedings. 
[4] A. Makovec et al., Radiation hard polyimide-coated FBG optical sensors for relative humidity monitoring in the CMS experiment at CERN, JINST 9, C03040 (2014).

[5] B. Francois, Offline performance of the CMS Tracker during early Run II, EPS-HEP 2015 proceedings. 